\title{
BNT162b2 and ChAdOx1 SARS-CoV-2 Post-vaccination Side-Effects Among Saudi Vaccinees
}

\author{
Ahmed N. Alghamdi ${ }^{\dagger}$, Mohammed I. Alotaibi ${ }^{\dagger}$, Adel S. Alqahtani, Daifullah Al Aboud and \\ Ahmed S. Abdel-Moneim*
}

College of Medicine, Taif University, Taif, Saudi Arabia

\section{OPEN ACCESS}

Edited by:

Reza Lashgari,

Shahid Beheshti University, Iran

Reviewed by:

Shyh Poh Teo,

Raja Isteri Pengiran Anak Saleha

Hospital, Brunei

Flor Pujol,

Instituto Venezolano de

Investigaciones Cientificas

(IVIC), Venezuela

AbdulAzeez Adeyemi Anjorin,

Lagos State University, Nigeria

Andrew Clark,

University of Texas Southwestern

Medical Center, United States

*Correspondence:

Ahmed S. Abdel-Moneim

asa@tu.edu.sa;

asa@bsu.edu.eg

tThese authors have contributed equally to this work

Specialty section:

This article was submitted to Infectious Diseases - Surveillance,

Prevention and Treatment,

a section of the journal

Frontiers in Medicine

Received: 17 August 2021 Accepted: 13 September 2021

Published: 08 October 2021

Citation:

Alghamdi AN, Alotaibi MI,

Alqahtani AS, Al Aboud D and Abdel-Moneim AS (2021) BNT162b2

and ChAdOx1 SARS-CoV-2

Post-vaccination Side-Effects Among

Saudi Vaccinees.

Front. Med. 8:760047.

doi: 10.3389/fmed.2021.760047
Background: Vaccination against SARS-CoV-2 is important for reducing hospitalization and mortalities. Both Pfizer-BioNTech (BNT162b2) and the Oxford-AstraZeneca (ChAdOx1 nCoV-19) vaccines are used in Saudi Arabia and in many parts of the world. Post-vaccinal side effects were recorded, so we aimed to screen different complaints after vaccination among vaccinees in Saudi Arabia.

Methods: An online questionnaire was designed to screen the local, systemic, and allergic post vaccination reactions for vaccinees who received either one or two doses of the BNT162b2 vaccine or one dose of the ChAdOx1 vaccine. The number and percentage were recorded for each response and analyzed using cross-tab and Chi square tests. The degree of the severity of post vaccination reactions were analyzed using Roc curve. The cofactors that may affect the severity of post-vaccinal reactions including previous COVID-19 infection, age, sex, body mass index, and comorbidities were investigated.

Results: During our study, 4,170 individuals reported their responses: 2,601 received one dose of BNT162b2, of whom 456 completed the second dose, and 1,569 received a single dose of ChAdOx1. The side effects were reported in $85.6 \%$ of BNT162b2 vaccinees and $96.05 \%$ of ChAdOx1 vaccinees who voluntarily responded to a survey about post-vaccination side effects. The side effects were more severe in BNT162b2 than ChAdOx1. ChAdOx1 vaccinees reported mild, moderate, severe and critical side effects in $30.13,28.62,29.73$, and $1.53 \%$, respectively. In contrast, mild side effects were recorded among the majority of BNT162b2 vaccinees (63.92\%) while moderate, severe, and critical side effects were $27.67,7.68$, and $0.72 \%$, respectively. Both local and systemic side effects were recorded more frequently in ChAdOx1 in comparison to BNT162b2 vaccinees. Palpitation was among the new systemic side effects reported in the current study in high frequency. Abnormal menstrual cycle (delaying/increase hemorrhages or pain) was also reported in $0.98 \%$ (18/1846) of Pfizer-BioNTech and $0.68 \%(7 / 1028)$ of $\mathrm{ChAdO} \times 1$ vaccinees, while deep vein thrombosis was only reported in a single case vaccinated with BNT162b2 vaccine.

Conclusion: Both vaccines induced post-vaccinal side effects; however, ChAdOx1 induces a higher frequency of post-vaccinal systemic side effects than BNT162b2.

Keywords: COVID-19, SARS-CoV-2, COVID vaccines, post vaccination side effects, ChAdOx1 nCoV-19 vaccine, BNT162b2 mRNA COVID-19 vaccine, palpitation, menstrual cycle disturbance 


\section{BACKGROUND}

The SARS-CoV-2 pandemic continues to spread worldwide, following its first emergence in December 2019. The virus induces coronavirus disease (COVID-19) that might be asymptomatic or mild in the majority of patients; however, $20 \%$ of infected patients are prone to develop severe to serious diseases with fatal consequences (1). Old age, gender, asthma, hypertension, and other cardiovascular diseases are among the risk factors of COVID-19 (2-4).

Vaccination is the most successful way to prevent the spread of many life-threatening infectious diseases. Thanks to vaccines, millions of lives are saved annually against more than 20 infectious diseases. Some countries have implemented local legislations for COVID-19 obligatory vaccination for access to private and governmental public sectors, making it mandatory for travelers prior to entry into such countries. Despite the significant number of vaccinated individuals worldwide, many people around the world are reluctant to get vaccinated against COVID-19. This fact could be due to the widespread rumors regarding the panic from the side effects that have led to a lack of trust in COVID-19 vaccines.

Starting from December 2020, many SARS-CoV-2 vaccines have been released for emergency use, including spike- gene RNA-vaccines encapsulated in lipid nanoparticles, such as BNT162b2 (Pfizer-BioNTech) and mRNA-1273 (Moderna); chimpanzee adenovirus vector vaccine harboring SARS-CoV$2 \mathrm{~S}$ gene (ChAdOx1- AstraZeneca), human adenovirus 26 (Ad26.COV2.S-Johnson \& Johnson/Janssen), and inactivated SARS-CoV-2 vaccines (Sinopharm and Sinovac). Saudi Arabia approved BNT162b2 then ChAdOx1 for use in the vaccination protocol on 10 December 2020, and 18 February 2021, respectively. Both vaccines have proven to be safe and efficacious as evidenced by the results of clinical trials $(5,6)$. There is a need of up to 14 billion doses of COVID-19 vaccines to cover $70 \%$ of global coverage (7). This fact results in the recommendation of the WHO to temporary halt of giving the second dose of COVID19 vaccine, with the aim of covering more unvaccinated people worldwide (8).

Pain, redness, swelling, fever, tiredness, headache, muscle pain, chills, and nausea are among the common side effects of the vaccines (9). Most of such side effects disappear within 2 days after vaccination (9). Other rare side effects, including vesicolous-bullous skin (10), herpes simplex or varicella zoster reactivation (11-13), nephrotic syndrome and acute kidney injury (14) were also reported. Meanwhile, serious post-vaccinal reactions, including intravascular thrombosis and thrombocytopenia are associated with both ChAdOx1 and the BNT162b2 vaccines; however, the frequency was 5-fold higher in ChAdOx1 than in BNT162b2 (15). It was found that the ChAdOx1 vaccine but not BNT162b2 enhances the production of anti-platelet factor 4 antibodies $(15,16)$. Accordingly, some countries in Europe suspended the use of the ChAdOx1 vaccine. However, KSA and other European countries decided to continue using the vaccine according to the WHO recommendation that benefits of using ChAdOxl vaccine outweigh its risks (17).
On the other hand, anaphylaxis reaction has been reported among BNT162b2 vaccinees in an approximate rate of 1:200,000. Such reaction was most probably induced by the polyethylene glycol (PEG $2000 \mathrm{Da}$ ) (18). Interestingly ChAdOx1 vaccine contains polysorbate 80 , a derivative of the PEG, which is used in a similar concentration in many vaccines including DTaP, HB vaccine, HPV, and influenza vaccines (19). The relatively lower molecular weight of the polysorbate $80(1310 \mathrm{Da})$ in comparison to PEG $(2000 \mathrm{Da})$ and the fact that polysorbate 80 is being used in many vaccines, monoclonal antibodies, and most of the injectable biological reagents for many decades alleviates its role as a possible trigger of anaphylaxis $(18,19)$.

SARS-CoV-2 vaccines are successful in reducing the severity of the disease, hospitalization, and mortality (20). The safety of SARS-CoV-2 vaccines has been successfully and carefully monitored prior to their authorization. However, the tracking of side effects that were not detected during clinical trials is still needed. The current study aimed to screen the incidence of local, systemic, and allergic reactions following SARS-CoV2 vaccination. We also intended to screen possible factors that may affect the incidence and severity of post-vaccinal reactions, including previous COVID-19 infection, age, sex, and other immunocompromising conditions.

\section{METHODS}

\section{Participants}

Vaccination was an inclusion criterion for filling the questionnaire. The questions seek the following information: whether participants are suffering from any chronic diseases or immunosuppressive disorders, whether they suffered from a previous SARS-CoV-2 infection or not, and the type and number of doses of the vaccine. The questionnaire included a leading question asked about the presence of absence of the side effects following vaccination. In case they have symptoms, they were asked about the individual side effects they experienced after the first and the second doses of the vaccines in separate sets of questions. There were three main clusters of questions including those related to local, allergic, and systemic reactions in addition to other unlisted symptoms. A question about the intensity of reaction or the severity of side effects was included; it graded the severity into mild (minor reactions that extended for $\leq 1-2$ days), moderate (tolerable reactions extended to 2-3 days), severe (exacerbation of the post-vaccinal reactions in barely tolerable manner extended for $\geq 3$ days but did not require hospitalization), and critical (adverse signs were severe enough to require hospitalization). We also asked about the duration of the post-vaccination side effects till the 7 th day post vaccination and provided an option if the signs continued for more than 7 days. Also included was an open question so the respondent could write any other sign not listed in the questions. A link to the online questionnaire was distributed in different social media including Twitter, Snapchat, and WhatsApp.

\section{Statistical Analysis}

The differences between the two vaccinated groups (BNT162b2 vs. those who received ChAdOx1) were compared using Chi 
TABLE 1 | Frequencies and characteristics of the subjects in the current study.

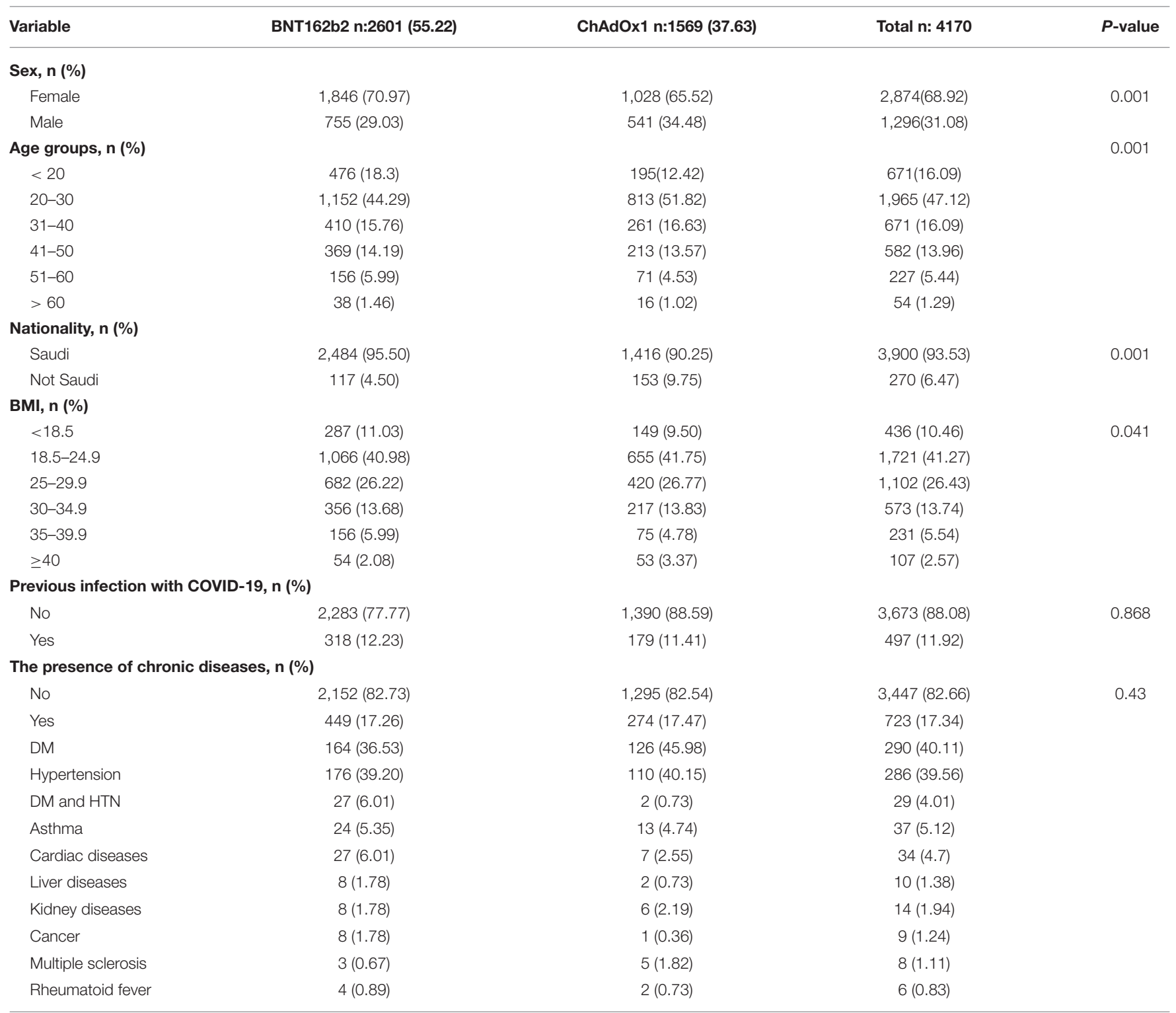

Data are showed as number (percentage). Chi-square test was used to calculate the p-value for the difference between the first dose of BNT162b2 and ChAdOx1 vaccines.

square and Mann Whitney tests. The differences between the severity of the post-vaccinal reaction after the first and second BNT162b2 vaccination doses were also compared. The percentage of vaccinees experiencing side effects after having received the vaccine were calculated. All vaccinees were included in the adverse effects' analysis, including those who received a single dose of BNT162b2 or ChAdOx1. The probability of having severe side effects following the first and second BNT162b2 doses were also compared. Roc curve and logistic regressions were used for each of the specified variables to investigate whether adverse effects varied among different groups, and whether the previous exposure to COVID-19 infection could alter the response. Area under the curve $(A U C) \leq 0.5$ poor discrimination or not useful, $0.7-0.8$ is considered a good or an acceptable value, while $0.8-0.9$ is very good value, while $\geq 0.9$ is an excellent $(21,22)$.

\section{RESULTS}

\section{Demographic Characteristic of the Studied Subjects}

The total number of users who reported vaccination with the BNT162b2 vaccine (2,601:1,846 females and 755 males) were higher than those who reported getting vaccinated with ChAdOx1 (1,569:1,028 females and 541 males). This included slightly younger individuals and they were more frequently female than users who reported receiving the ChAdOx1 inoculation (Table 1). A majority of respondents were 20-30 years old (44.29\% (1152/2601), 51.8\% (813/1569) for BNT162b2 and ChAdOx1, respectively). The study found a small number of respondents above 50 years old; $7.46 \%$ (194/2601) and 5.54\% (87/1569), for BNT162b2 and ChAdOx1, respectively (Table 1). 
TABLE 2 | Correlation of the severity of the post-vaccinal reactions and different parameters.

\begin{tabular}{|c|c|c|c|c|c|c|}
\hline Variable & Don't have side effect & Mild & Moderate & Severe & Critical & $\begin{array}{l}\text { Significance } \\
\text { and correlation }\end{array}$ \\
\hline \multicolumn{7}{|l|}{ Sex, n (\%) } \\
\hline Male & $210(16.2)$ & $615(47.5)$ & $311(24.0)$ & $148(11.4)$ & $12(0.9)$ & $R=-0.133$ \\
\hline \multicolumn{7}{|l|}{ Age groups, n (\%) } \\
\hline$<20$ & $82(12.2)$ & 277 (41.3) & 207 (30.8) & $96(14.3)$ & $9(1.3)$ & $P<0.016$ \\
\hline $31-40$ & 69 (10.3) & $316(47.1)$ & $177(26.4)$ & 105 (15.6) & $4(0.6)$ & \\
\hline $41-50$ & $69(11.9)$ & 283 (48.6) & $143(24.6)$ & 79 (13.6) & $8(1.4)$ & \\
\hline $51-60$ & $41(18.1)$ & 105 (46.3) & $58(25.6)$ & $21(9.3)$ & $2(0.9)$ & \\
\hline$>60$ & 18 (33.3) & $22(40.7)$ & $9(16.7)$ & $4(7.4)$ & $1(1.9)$ & \\
\hline \multicolumn{7}{|l|}{ Nationality, n (\%) } \\
\hline Saudi & $410(10.5)$ & $1,776(45.5)$ & $1,113(28.5)$ & $565(14.5)$ & $36(0.9)$ & $P<0.016$ \\
\hline $18.5-24.9$ & $162(9.4)$ & $744(43.2)$ & $520(30.2)$ & $282(16.4)$ & $13(0.8)$ & $R=-0.040$ \\
\hline 25-29.9 & $133(12.1)$ & $500(45.4)$ & $317(28.8)$ & $140(12.7)$ & $12(1.1)$ & \\
\hline $30-34.9$ & $56(9.8)$ & $274(47.8)$ & $149(26.0)$ & $89(15.5)$ & $5(0.9)$ & \\
\hline $35-39.9$ & 29 (12.6) & $121(52.4)$ & $55(23.8)$ & $24(10.4)$ & $2(0.9)$ & \\
\hline$\geq 40$ & $15(14.0)$ & 41 (38.3) & $32(29.9)$ & $16(15.0)$ & $3(2.8)$ & \\
\hline \multicolumn{7}{|l|}{ Type of the vaccine } \\
\hline BNT162b2 (n:2601) & $375(14.42)$ & $1,423(54.71)$ & $616(23.68)$ & $171(6.57)$ & $16(0.62)$ & $P<0.01$ \\
\hline ChAdOx1 $(n=1,569)$ & $62(3.95)$ & $454(28.93)$ & $582(37.09)$ & $448(28.55)$ & $23(1.47)$ & $R=0.381$ \\
\hline \multicolumn{7}{|c|}{ Previous infection with COVID-19, n (\%) } \\
\hline No & $385(10.5)$ & $1,681(45.8)$ & $1,040(28.3)$ & $528(14.4)$ & $39(1.1)$ & $P<0.016$ \\
\hline Yes & $52(10.5)$ & $196(39.4)$ & $158(31.8)$ & $91(18.3)$ & $0(0.0)$ & $R=0.035$ \\
\hline
\end{tabular}

\section{Correlation Between the Severity of Post-vaccinal Reaction and Different Parameters}

We studied the correlation between the severity of the postvaccinal reactions and age, sex, nationality, body mass index, previous COVID-19 infection, and the presence of chronic diseases in the vaccinated subjected. The presence of chronic diseases was found to show significant correlation to the development of post-vaccinal adverse effects $(P<0.016, R$ $=0.2$ ). The female gender was also associated with the development of moderate and severe post-vaccinal adverse effects $(P<0.015, R=-0.133)$ (Table 2). There is a significant correlation between the type of vaccine and the severity of the post-vaccinal reaction $P>0.01, R=0.381$. The severe post vaccination side effects were found to be correlated with ChAdOx1 vaccine (Table 2). The Roc curve of the ChAdOx1 vaccine showed an acceptable good AUC value, 0.713 , with the level of disease severity, 0.008 standard error and $P<0.001$. While a single dose or two doses of BNT162b2 showed poor AUC of 0.347 and 0.378 , respectively (Figure 1).

\section{Post-vaccinal Adverse Effects Following Administration of BNT162b2 or ChAdOx1}

The severity of post-vaccinal side effects was found to be significantly higher in females in comparison to males (Table 3 ). ChAdOx1 vaccinees reported mild, moderate, severe, and critical side effects in 454/1569 (30.13\%), 582/1569 (28.62\%), 448/1569 $(29.73 \%)$, and $23 / 1569(1.53 \%)$, respectively. In contrast, mild side effects were recorded among the majority of BNT162b2 vaccinees 1423/2601 (63.92\%) while moderate, severe, and critical side effects were 616/2601 (27.67\%), 171/2601 (7.68\%) and 16/2601 (0.72\%), respectively (Table 4).

Most respondents to this survey about vaccine side effects suffered from one or more side effects while only $14.42 \%$ (375/2601) and 3.95\% (62/1569) did not record any side effect for BNT162b2 and ChAdOx1, respectively. The most common side effect was localized pain after vaccine injection: 2,088 (80.27\%), $1,347(85.85 \%)$ for BNT162b2 and ChAdOx1, respectively. For ChAdOx1, fatigue 1,354 (86.30\%), muscle pain 1,100 (70.11\%), and headache were the common complaints. Meanwhile, for BNT162b2, fatigue 1,358 (52.21\%), muscle pain 1,088 (41.83\%), and headache were also the common systemic complaints but 


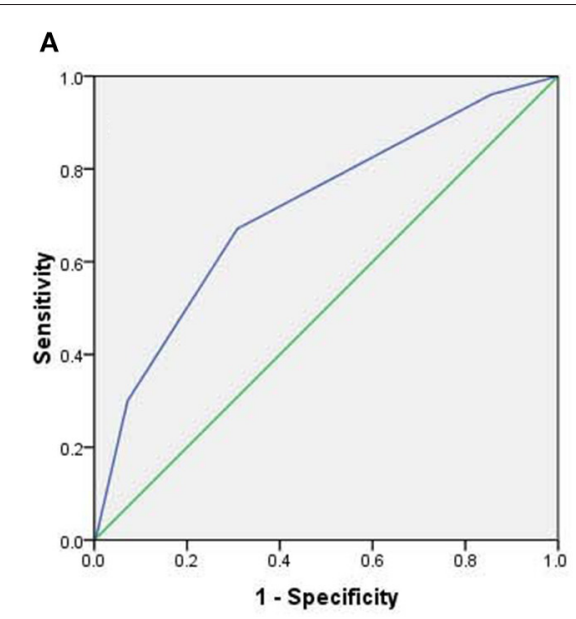

B

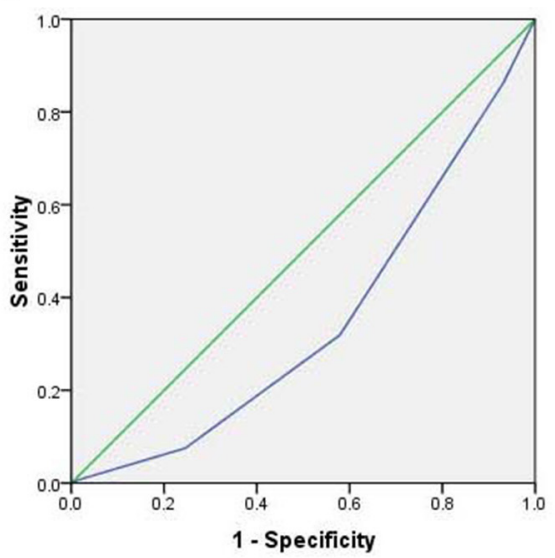

C

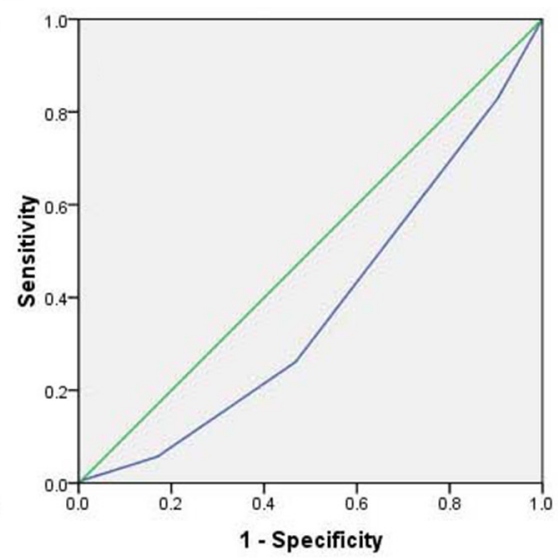

FIGURE 1 | The Roc curve demonstrates the level of severity of post-vaccinal side effects and type of the vaccines. (A) ChAdOx1 vaccine, (B) A single dose of BNT162b2 vaccine, (C) Two doses of BNT162b2 vaccine.

were significantly lower than that detected for the ChAdOx1 vaccine. Insomnia 430 (27.40\%), palpitation 269 (17.14\%), chest pain 209 (13.32\%), breathlessness 153 (9.75\%), diarrhea 151 (9.62), and abdominal pain 149 (9.50) were recorded in

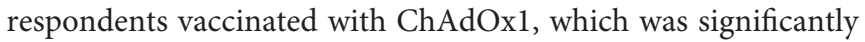
higher than those reported for BNT162b2 (Table 4). The detected palpitation was not correlated to any of the reported chronic diseases related to cardiac diseases (Table 5). Fever, chills, palpitation, joint pain, and impaired concentration showed a highly significant variation among subjects vaccinated with ChAdOx1 ( $\sim 2$-fold or more of the percentage detected following BNT162b2 vaccination; Table 4). Other side effects, including allergies (itching, rashes, and swollen lips) were reported in both BNT162b2 and ChAdOx1 vaccinated subjects but there were no significant differences among the two groups. There were no significant differences between the vaccinees with regard to the allergic or local side effects. ChAdOx1 showed a higher percentage of local pain at the site of injection than BNT162b2. In contrast, subjects vaccinated with ChAdOxl showed a highly significant increase in the duration of systemic reactions than those vaccinated with BNT162b2. About 11.96\% (304/2601) and $13.7 \%(215 / 1569)$ showed side effects extended from 4->7 days, while $72.9 \%(1897 / 2601)$ and $82.1 \%$ (1288/1569) showed side effects extended from 1-3 days for BNT162b2 and ChAdOx1, respectively (Table 4 ).

\section{Post-vaccinal Adverse Effects Following Administration of First and Second Doses of BNT162b2}

Interestingly, subjects who received the second dose of BNT162b2 showed more moderate and severe reactions in a highly significant manner in comparison to the same subjects after the first dose of the vaccine $(P<0.001)$. No significant variation in local pain or post-vaccinal allergic reactions or even the duration of side effects was detected among respondents got the first and second dose of BNT162b2. Significant systemic reaction variations were more pronounced among subjects after getting the second dose in comparison to the same subjects after getting the first dose of the vaccine (Table 4). In contrast, the complaint of joint and muscle pain was more frequently detected after the first dose, as compared to the second dose. Diarrhea $(308 / 456,67.84 \%)$ and muscle pain $(162 / 456,70.11 \%)$ were very prominent complaints in a majority of the subjects who received the second dose in comparison to those got the first dose (19/456, $4.17 \%)(31 / 456,6.83 \%)$, respectively (Table 4).

\section{Uncommon Post-vaccinal Adverse Effects Added by the Participants}

The studied subjects reported uncommon post-vaccinal adverse effects, with a relatively considerable number of abnormal menstrual cycle, including increase in the time or increase in the pain or the bleeding, which was higher in BNT162b2 (18 cases) than in ChAdOx1 (7 cases). Other recorded signs included sore throat and/or dry mouth in 12 cases vaccinated with BNT162b2 and 3 cases vaccinated with ChAdOx1. Other rare signs were reported: anxiety, depression, sleepiness, mood disturbance, numbness of face, bruises in the leg, deep vein thrombosis, hypertension, pain in the testes, pain in the eyes, blurred vision, pain in the ear, and tinnitus (Table 6).

\section{DISCUSSION}

In this study, the majority of respondents were 20-30 years old for both BNT162b2 and ChAdOx1. Considerable adverse side effects among respondents under 50 years of age, were reported. Previous studies support our findings as they showed that a higher rate of post-vaccinal side effects occurred among young subjects (23-25).

Subjects who suffered from chronic diseases are assumed to possess a weakened responsiveness to immunogens and hence are assumed to experience reduced side effects following vaccines $(26,27)$. In this study, chronic diseases were found to show significant correlation to the development of post-vaccinal 
TABLE 3 | The post vaccine adverse effects after vaccination in male and female subjects.

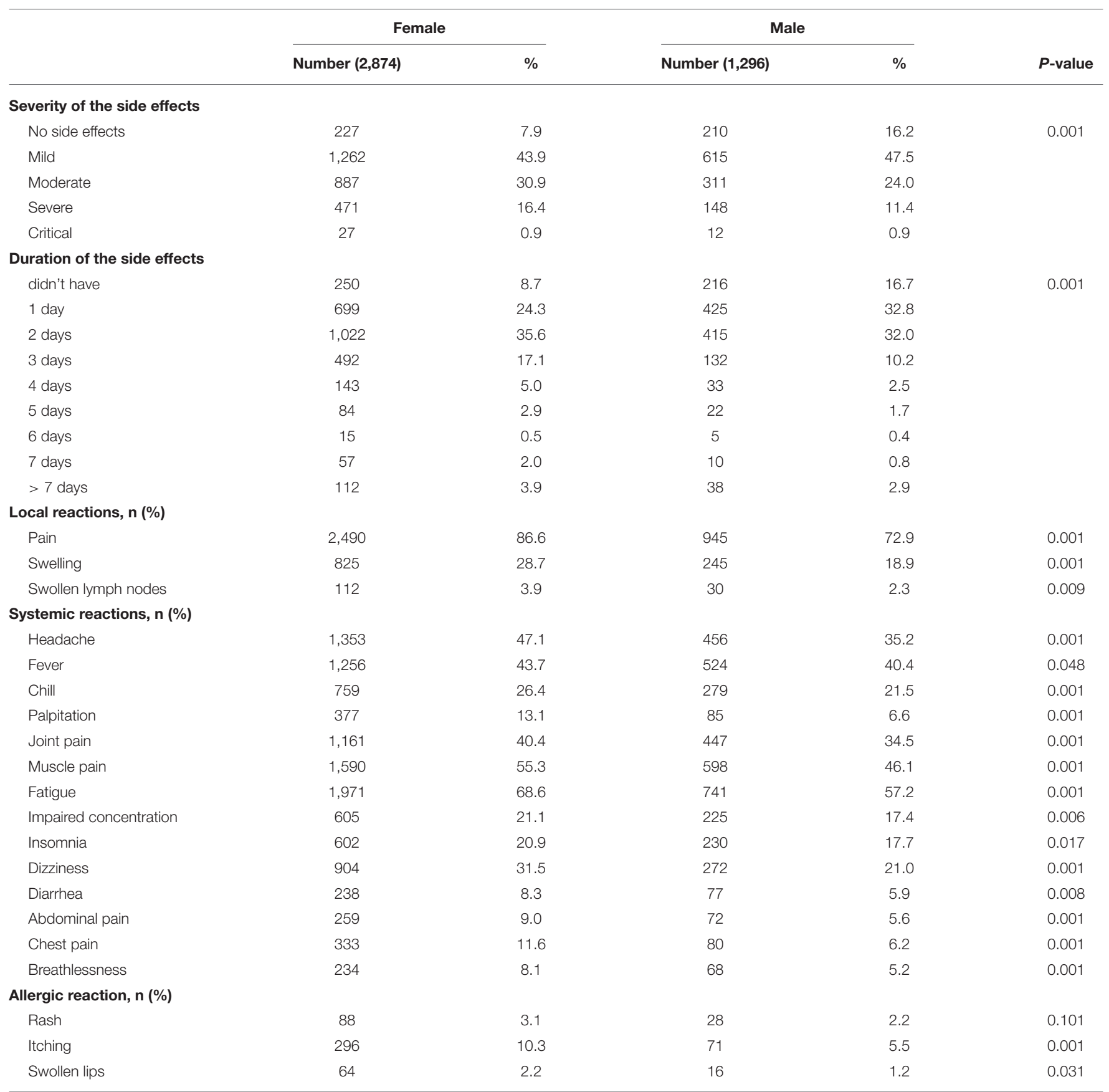

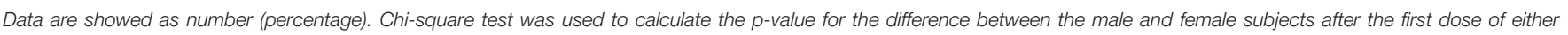
BNT162b2 or ChAdOx1.

adverse effects. However, no published information is available regarding these clusters of patients and their responsiveness or side effects following different COVID-19 vaccinations.

Subjects who previously experienced COVID-19 infection were reported to develop more severe side effects after vaccination (28). However, in our study, we did not find a considerable correlation between previous SARS-CoV-2 infection and the severity of post-vaccinal side effects.
In this study, the female gender was also associated with development of moderate and severe post-vaccinal adverse effects, showing a positive correlation of gender to the severity of the post-vaccinal side effects. This finding also agrees with other findings and confirms that the development of post-vaccinal side effects were significantly higher in females than in males $(23,24,29)$. However, it disagrees with a recent study (30) that reported that males 
TABLE 4 | Comparison between the post vaccine adverse effects after vaccination with BNT162b2 and ChAdOx1 as well as those after the first and the second doses of BNT162b2.

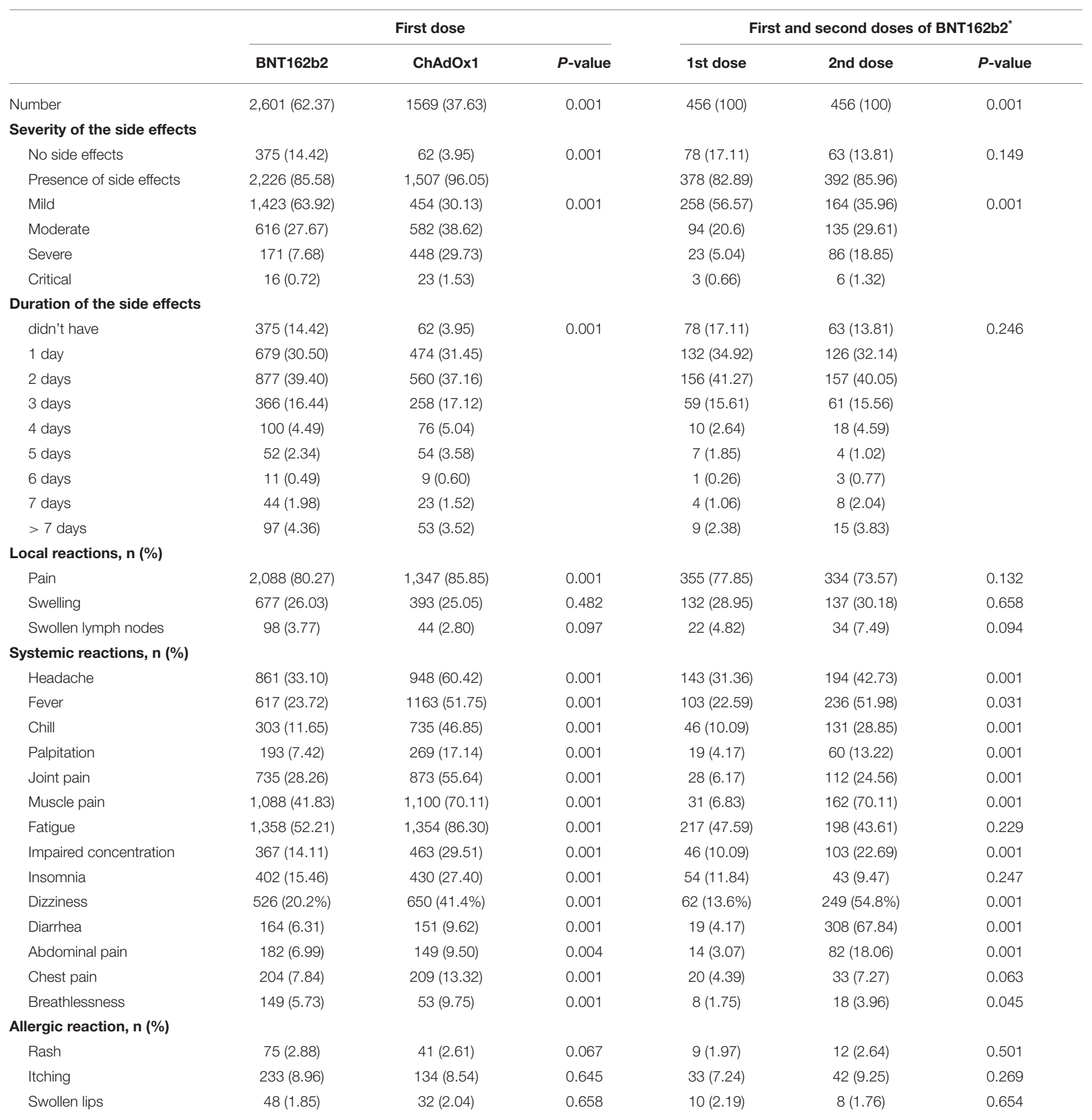

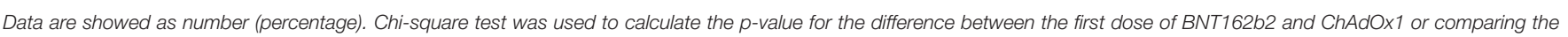
first dose of BNT162b2 with the second dose.

*The people who got the second dose of the vaccine $(n=456)$ were extracted from the total number of Pfizer-BioNTech vaccinees $(n=2601)$.

developed more severe post-vaccinal reactions in comparison to females.

In general, ChAdOx1 showed a highly significant increase in the severity and duration of systemic post-vaccinal reactions than
BNT162b2. Meanwhile, the side effects of BNT162b2 after the second dose were more severe than that reported after the first dose of the vaccine. In this respect, our findings agree with other studies that reported similar findings $(24,31,32)$. 
TABLE 5 | Relationship between palpitation and the presence of hypertension, and cardiac disease in the tested subjects.

\begin{tabular}{lcccc}
\hline & & \multicolumn{2}{c}{ Palpitation } & $\begin{array}{c}\text { Significance and } \\
\text { correlation }\end{array}$ \\
\cline { 3 - 4 } & & No & Yes & \\
\hline HTN & No & 3,443 & 441 & $P<0.97$ \\
Cardiac disease & Yes & 265 & 21 & $R=0.015$ \\
HTN and DM & No & 3,678 & 458 & $P<0.636$ \\
& Yes & 30 & 4 & $R=0.007$ \\
& No & 4,220 & 461 & $P<0.216$ \\
& Yes & 28 & 1 & $R=0.022$ \\
\hline
\end{tabular}

TABLE 6 | Uncommon post-vaccinal adverse effects reported by the participants.

\begin{tabular}{lcccc}
\hline & \multicolumn{2}{c}{ BNT162b2 } & & ChAdOx1 \\
\cline { 2 - 3 } \cline { 5 - 5 } & 1st dose N (\%) & 2nd dose N (\%) & 1st dose N (\%) \\
\hline Abnormal menstrual cycle & $18(0.69)$ & $1(0.22)$ & & $7(0.45)$ \\
Anxiety & $1(0.04)$ & $0(0.00)$ & & $1(0.06)$ \\
Depression & $2(0.08)$ & $0(0.00)$ & & $1(0.06)$ \\
Mood disturbance & $2(0.08)$ & $0(0.00)$ & & $1(0.06)$ \\
Numbness of face & $1(0.04)$ & $0(0.00)$ & & $0(0.00)$ \\
Sore throat/dry mouth & $12(0.46)$ & $3(0.66)$ & & $3(0.19)$ \\
Bruises in the leg & $2(0.08)$ & $0(0.00)$ & & $1(0.06)$ \\
Deep venous thrombosis & $1(0.04)$ & $0(0.00)$ & & $0(0.00)$ \\
Hypertension & $2(0.08)$ & $3(0.66)$ & & $0(0.00)$ \\
Pain in testis & $0(0.00)$ & $1(0.22)$ & & $1(0.06)$ \\
Pain in eyes & $4(0.15)$ & $0(0.00)$ & & $2(0.13)$ \\
Blurred vision & $1(0.04)$ & $0(0.00)$ & & $1(0.00)$ \\
Pain in ear & $1(0.04)$ & $1(0.22)$ & & $1(0.06)$ \\
Tinnitus & $0(0.00)$ & $0(0.00)$ & $2(0.13)$ \\
\hline
\end{tabular}

The most common side effect was local pain after vaccine injection: $80.27 \%$ and $85.85 \%$ for BNT162b2 and ChAdOx1, respectively. This finding agrees with the data of phase $2 / 3$ clincal trial that reported $88 \%$ pain at the site of injection following vaccination with ChAdOx1 (25), in individuals 18-55 of age and it reached $>80 \%$ in individuals ( $\geq 16$ years old) vaccinated with BNT162b2 (23). Similarly, the detected fatigue, muscle and joint pain following BNT162b2 and ChAdOx1 vaccinations matched what was reported previously $(23,25)$.

In the USA, many neurological side effects were reported $(33,34)$. In the current study, signs of transient central nervous system affections ranged from 14.1 to $29.5 \%$ of the reported side effects. They included impaired concentration, insomnia, and dizziness. These affections were reported in a significantly higher frequency in ChAdOx1 as compared to BNT162b2. Similarly, their frequencies were higher after the second dose of BNT162b2, in comparison to those after the first dose. Meanwhile, rare solitary neurological signs, including anxiety, depression, sleepiness, mood disturbance, numbness of face, pain in the ear, and tinnitus were also detected in the current study. Our finding agrees with previous reports of rare reports of tremor, diplopia, dysphonia, seizures, tinnitus, herpes reactivation, facial palsy, transverse myelitis, and acute disseminated encephalomyelitis detected in the USA following COVID-19 vaccination $(33,34)$.

Palpitation was not previously reported among the side effects of COVID-19 vaccination; however, a case report of postural orthostatic tachycardia syndrome was recently reported following BNT162b2 vaccination (35). Interestingly, in the current study, palpitation was reported with high frequency after COVID-19 vaccination: $7.42 \%$ and $17.14 \%$ for BNT162b2 and ChAdOx1, respectively. Similarly, the signs were reported more frequently in vaccinees after the second dose $(13.22 \%)$ in comparison to the first dose (4.17\%) for BNT162b2. In addition, chest pain and breathlessness were also reported in this study with ChAdOx1 and to a lower frequency among BNT162b2 vaccinees. In April 2021, the Vaccine Adverse Event Reporting System (VAERS) detected more than 1,000 cases reporting myocarditis and pericarditis following mRNA vaccination (36). Palpitation was also reported in another study in Saudi Arabia in $7(0.4 \%)$ subjects following vaccination with ChAdOx1 (30) and in South Korea in $28.3 \%$ and $4.3 \%$ of ChAdOx1 and NT162b2 vaccinees, respectively (37).

Diarrhea and abdominal pain were recorded in respondents vaccinated with ChAdOxl. The percentages of such side effects were significantly higher than those reported for BNT162b2. The latter showed a higher frequency of complaints after the second dose in comparison to the first dose. A meta-analysis study reported similar side effects for both BNT162b2 and ChAdOx1 (38).

Menstrual disturbance, including excessive hemorrhage and irregular menstrual cycle were reported after the administration of BNT162b2 (643 cases) and ChAdOx1 (315 cases) (39). In contrast, in this study, irregular menstrual cycle was reported in 18 cases vaccinated with BNT162b2 (0.69\%) and only 7 cases $(0.45 \%)$ vaccinated with ChAdOx1.Thrombocytopenia and underlying platelets disorders, or possible hormonal disturbance may explain excessive menstrual bleeding. More investigations are needed to determine the link between COVID-19 vaccination and the menstrual irregularities $(40,41)$.

\section{CONCLUSION}

In conclusion, both vaccines induce transient side effects that ranged from mild to severe. ChAdOx1 induced more severe side effects than BNT162b2. The latter vaccine induces more severe adverse effects after the second dose of the vaccine. High frequency of palpitation was reported following vaccination. All the reported side effects are tolerable. Women are more prone to developing more severe side effects and for longer durations. Palpitation is among the common systemic side effects that appear following vaccination of both vaccines.

\section{Limitation of the Study}

The results of this questionnaire are self-reported from those receiving the vaccine and not clinically confirmed by physicians 
as it may impact result reporting due to differences in interpretation and tolerance thresholds from patient to patient. The study is limited by possible information bias, including misclassification and the duration description of some important manifestations like palpitation and chest pain. The utilization of social media may bias the cohort toward age demographics most familiar with the technology (as evidenced by the high numbers of 20-30-year-olds and a lack of 50+ year old patients) and socioeconomic demographics with routine access to such platforms.

\section{DATA AVAILABILITY STATEMENT}

The original contributions presented in the study are included in the article/supplementary material, further inquiries can be directed to the corresponding author/s.

\section{REFERENCES}

1. Veiga VC, Prats JGG, Farias DLC, Rosa RG, Dourado LK, Zampieri FG, et al. Effect of tocilizumab on clinical outcomes at 15 days in patients with severe or critical coronavirus disease 2019: randomised controlled trial. BMJ. (2021) 372:n84-n84. doi: 10.1136/bmj.n84

2. Berlin DA, Gulick RM, Martinez FJ. Severe Covid-19. N Engl J Med. (2020) 383:2451-60. doi: 10.1056/NEJMcp2009575

3. Williamson EJ, Walker AJ, Bhaskaran K, Bacon S, Bates C, Morton CE, et al. Factors associated with COVID-19-related death using OpenSAFELY. Nature. (2020) 584:430-6. doi: 10.1038/s41586-020-2521-4

4. Wu C, Chen X, Cai Y, Xia J, Zhou X, Xu S, et al. Risk factors associated with acute respiratory distress syndrome and death in patients with coronavirus disease 2019 Pneumonia in Wuhan, China. JAMA Intern Med. (2020) 180:934-43. doi: 10.1001/jamainternmed.2020. 0994

5. Folegatti PM, Ewer KJ, Aley PK, Angus B, Becker S, Belij-Rammerstorfer $\mathrm{S}$, et al. Safety and immunogenicity of the ChAdOxl nCoV-19 vaccine against SARS-CoV-2: a preliminary report of a phase $1 / 2$, single-blind, randomised controlled trial. Lancet. (2020) 396:467-78. doi: 10.1016/S0140-6736(20)31604-4

6. Alley SJ, Stanton R, Browne M, To QG, Khalesi S, Williams SL, et al. As the pandemic progresses, how does willingness to vaccinate against COVID-19 evolve? Int J Environ Res Public Health. (2021) 18:797. doi: 10.3390/ijerph18020797

7. Kim J. COVID-19 vaccines: taking a shot beyond efficacy. In: SöderlundVenermo M, Varma A, Pujol F, Venter M, Nevels MM, Kuhn R, et al. editors. The First Meeting of the World Society for Virology | Tackling Global Viral Epidemics. (2021).

8. WHO. Recommendation of temporary halt of giving the booster dose of COVID-19 vaccine. Nature. (2021) 596:317. doi: 10.1038/d41586-021-02219-w

9. CDC. Possible Side Effects After Getting a Covid-19 Vaccine. (2021). Available online at: https://www.cdc.gov/coronavirus/2019-ncov/vaccines/expect/after. html (accessed August 6, 2021).

10. Coto-Segura P, Fernández-Prada M, Mir-Bonafé M, García-García B, González-Iglesias I, Alonso-Penanes P, et al. Vesicolous-bullous skin reactions induced by COVID-19 mRNA vaccine: report of four cases and review of the literature. Clin Exp Dermatol. (2021). doi: 10.1111/ced.14835. [Epub ahead of print].

11. Català A, Muñoz-Santos C, Galván-Casas C, Roncero Riesco M, Revilla Nebreda D, Solá-Truyols A, et al. Cutaneous reactions after SARS-COV-2 vaccination: A cross-sectional Spanish nationwide study of 405 cases. $\mathrm{Br} \mathrm{J}$ Dermatol. (2021). doi: 10.1111/bjd.20639. [Epub ahead of print].

\section{ETHICS STATEMENT}

The studies involving human participants were reviewed and approved by Taif University Ethical Committee with approval No. 42-169 on 3/05/2021.

\section{AUTHOR CONTRIBUTIONS}

ASA, ANA, MA, and DA collected, curated, analyzed the data, and wrote the first draft of the manuscript. AA-M conceptualized the study, verified the results, and critically revise the manuscript. All authors reviewed and edited revisions of the manuscript.

\section{FUNDING}

This work was funded by Taif University Deanship of Scientific Research (Project No. 1-441-41).

12. Marcantonio-Santa Cruz O, Vidal-Navarro A, Pesqué D, Giménez-Arnau AM, Pujol RM, Martin-Ezquerra G. Pityriasis rosea developing after COVID-19 vaccination. J Eur Acad Dermatol Venereol. (2021). doi: 10.1111/jdv.17498. [Epub ahead of print].

13. Psichogiou M, Samarkos M, Mikos N, Hatzakis A. Reactivation of varicella zoster virus after vaccination for SARS-CoV-2. Vaccines. (2021) 9:572. doi: 10.3390/vaccines9060572

14. Lebedev L, Sapojnikov M, Wechsler A, Varadi-Levi R, Zamir D, Tobar A, et al. Minimal change disease following the Pfizer-BioNTech COVID19 vaccine. Am J Kidney Dis. (2021) 78:142-5. doi: 10.1053/j.ajkd.2021. 03.010

15. Cari L, Fiore P, Naghavi Alhosseini M, Sava G, Nocentini G. Blood clots and bleeding events following BNT162b2 and ChAdOx1 nCoV-19 vaccine: An analysis of European data. J Autoimmun. (2021) 122:102685. doi: 10.1016/j.jaut.2021.102685

16. Greinacher A, Thiele T, Warkentin TE, Weisser K, Kyrle PA, Eichinger S. Thrombotic thrombocytopenia after ChAdOx1 nCov-19 vaccination. $N$ Engl J Med. (2021) 384:2092-101. doi: 10.1056/NEJMoa2104840

17. WHO. WHO Statement on AstraZeneca COVID-19 Vaccine Safety Signals. (2021). Available online at: https://www.who.int/news/item/17-03-2021who-statement-on-astrazeneca-covid-19-vaccine-safety-signals (accessed March 17, 2021).

18. Turner PJ, Ansotegui IJ, Campbell DE, Cardona V, Ebisawa M, El-Gamal Y, et al. COVID-19 vaccine-associated anaphylaxis: A statement of the World Allergy Organization Anaphylaxis Committee. World Allergy Organ J. (2021) 14:100517. doi: 10.1016/j.waojou.2021.100517

19. Banerji A, Wickner PG, Saff R, Stone CA, Robinson LB. mRNA vaccines to prevent COVID-19 disease and reported allergic reactions: current evidence and suggested approach. J Allergy Clin Immunol Pract. (2021) 9:1423-37. doi: 10.1016/j.jaip.2020.12.047

20. Swan DA, Bracis C, Janes H, Moore M, Matrajt L, Reeves DB, et al. COVID19 vaccines that reduce symptoms but do not block infection need higher coverage and faster rollout to achieve population impact. Sci Rep. (2021) 11:15531. doi: 10.1038/s41598-021-94719-y

21. Šimundić AM. Measures of diagnostic accuracy: basic definitions. EJIFCC. (2009) 19:203-11.

22. Mandrekar JN. Receiver operating characteristic curve in diagnostic test assessment. J Thorac Oncol. (2010) 5:1315-6. doi: 10.1097/JTO.0b013e3181ec173d

23. Polack FP, Thomas SJ, Kitchin N, Absalon J, Gurtman A, Lockhart S, et al. Safety and efficacy of the BNT162b2 mRNA Covid-19 vaccine. N Engl J Med. (2020) 383:2603-15. doi: 10.1056/NEJMoa2034577

24. Menni C, Klaser K, May A, Polidori L, Capdevila J, Louca P, et al. Vaccine sideeffects and SARS-CoV-2 infection after vaccination in users of the COVID 
symptom study app in the UK: a prospective observational study. Lancet. (2021) 21:939-49. doi: 10.1016/S1473-3099(21)00224-3

25. Ramasamy MN, Minassian AM, Ewer KJ, Flaxman AL, Folegatti PM, Owens DR, et al. Safety and immunogenicity of ChAdOx1 nCoV-19 vaccine administered in a prime-boost regimen in young and old adults (COV002): a single-blind, randomised, controlled, phase $2 / 3$ trial. Lancet. (2021) 396:197993. doi: 10.1016/S0140-6736(20)32466-1

26. O'brien KL, Finlay DK. Immunometabolism and natural killer cell responses. Nat Rev Immunol. (2019) 19:282-90. doi: 10.1038/s41577-019-0139-2

27. Berbudi A, Rahmadika N, Tjahjadi AI, Ruslami R. Type 2 diabetes and its impact on the immune system. Curr Diabetes Rev. (2020) 16:442-9. doi: 10.2174/1573399815666191024085838

28. Tissot N, Brunel AS, Bozon F, Rosolen B, Chirouze C, Bouiller K. Patients with history of covid-19 had more side effects after the first dose of covid-19 vaccine. Vaccine. (2021) 39:5087-90. doi: 10.1016/j.vaccine.2021. 07.047

29. Izumo T, Kuse N, Awano N, Tone M, Sakamoto K, Takada K, et al. Side effects and antibody titer transition of the BNT162b2 messenger ribonucleic acid coronavirus disease 2019 vaccine in Japan. Respir Investig. (2021) 59:635-42. doi: 10.1016/j.resinv.2021.06.003

30. Albahrani S, Albarra KA, Alghamdi OA, Abdullah Alghamdi M, Hakami FH, Abaadi AKA, et al. Safety and reactogenicity of the ChAdOx1 (AZD1222) COVID-19 vaccine in vaccinee in Saudi Arabia. Int J Infect Dis. (2021) 110:359-62. doi: 10.1016/j.ijid.2021.07.052

31. Alhazmi A, Alamer E, Daws D, Hakami M, Darraj M, Abdelwahab S, et al. Evaluation of side effects associated with COVID-19 vaccines in Saudi Arabia. Vaccines. (2021) 9:674. doi: 10.3390/vaccines 9060674

32. Andrzejczak-Grzadko S, Czudy Z, Donderska M. Side effects after COVID19 vaccinations among residents of Poland. Eur Rev Med Pharmacol Sci. (2021) 25:4418-21. doi: 10.26355/eurrev_202106_26153

33. Gee J. First month of COVID-19 vaccine safety monitoring-United States, December 14, 2020-January 13, 2021. MMWR Morb Mortal Wkly Rep. (2021) 70:7008e3. doi: 10.15585/mmwr.mm7008e3

34. Goss AL, Samudralwar RD, Das RR, Nath A. ANA investigates: neurological complications of COVID-19 vaccines. Ann Neurol. (2021) 89:856. doi: 10.1002/ana.26065

35. Reddy S, Reddy S, Arora M. A case of postural orthostatic tachycardia syndrome secondary to the messenger RNA COVID-19 vaccine. Cureus. (2021) 13:e14837. doi: 10.7759/cureus.14837
36. CDC. Myocarditis and Pericarditis Following mRNA COVID-19 Vaccination. (2021). Available online at: https://www.cdc.gov/coronavirus/2019-ncov/ vaccines/safety/myocarditis.html (accessed September 8, 2021).

37. Bae S, Lee YW, Lim SY, Lee JH, Lim JS, Lee S, et al. Adverse reactions following the first dose of ChAdOx1 nCoV-19 vaccine and BNT162b2 vaccine for healthcare workers in South Korea. J Korean Med Sci. (2021) 36:e115. doi: 10.3346/jkms.2021.36.e115

38. Pormohammad A, Zarei M, Ghorbani S, Mohammadi M, Razizadeh MH, Turner DL, et al. Efficacy and safety of COVID-19 vaccines: a systematic review and meta-analysis of randomized clinical trials. Vaccines. (2021) 9:467. doi: $10.3390 /$ vaccines 9050467

39. Merchant H. CoViD-19 post-vaccine menorrhagia, metrorrhagia or postmenopausal bleeding and potential risk of vaccine-induced thrombocytopenia in women. BMJ. (2021) 373:n958. doi: 10.1136/bmj.n958

40. Akiyama H, Kakiuchi S, Rikitake J, Matsuba H, Sekinada D, Kozuki $\mathrm{Y}$, et al. Immune thrombocytopenia associated with Pfizer-BioNTech's BNT162b2 mRNA COVID-19 vaccine. IDCases. (2021) 25:e1245. doi: 10.1016/j.idcr.2021.e01245

41. Razzaq AK, Al-Jasim A. Oxford-AstraZeneca coronavirus disease-2019 vaccine-induced immune thrombocytopenia on day two. Case Rep Hematol. (2021) 2021:2580832. doi: 10.1155/2021/2580832

Conflict of Interest: The authors declare that the research was conducted in the absence of any commercial or financial relationships that could be construed as a potential conflict of interest.

Publisher's Note: All claims expressed in this article are solely those of the authors and do not necessarily represent those of their affiliated organizations, or those of the publisher, the editors and the reviewers. Any product that may be evaluated in this article, or claim that may be made by its manufacturer, is not guaranteed or endorsed by the publisher.

Copyright (๑) 2021 Alghamdi, Alotaibi, Alqahtani, Al Aboud and Abdel-Moneim. This is an open-access article distributed under the terms of the Creative Commons Attribution License (CC BY). The use, distribution or reproduction in other forums is permitted, provided the original author(s) and the copyright owner(s) are credited and that the original publication in this journal is cited, in accordance with accepted academic practice. No use, distribution or reproduction is permitted which does not comply with these terms. 